\title{
VARIATION OF FIXATION DIRECTION WITH COLOUR OF FIXATION TARGET*
}

\author{
BY \\ D. H. FENDER
}

Reading

IN the course of recording eye movements it has become evident that the fixation direction of the eye changes slightly when the colour of the fixation target is changed. The technique used is that described by Ditchburn and Ginsborg (1953), photographing the movements of a beam of light reflected from a mirror attached to a contact lens worn by a subject. Several modifications as described by Fender (1955) have been introduced.

\section{Movement of Fixation Direction}

This effect has been studied in some detail and a typical set of results may be quoted for the right eye of Subject R.M.P.; if the fixation direction for a white target is taken as the origin of co-ordinates, then the fixation directions for various colours are given in the Table.

TABLE

\begin{tabular}{l|c|c}
\hline Colour & Horizontal (min. arc) & Vertical (min. arc) \\
\hline Blue & +0.3 & -1.2 \\
Green & 0 & -0.1 \\
Red & -12.0 & +3.6 \\
\hline+ indicates a nasal or upwards movement $\begin{array}{c}\text { indicates a temporal or downwards movement } \\
\text { Position of each fixation point } \pm 0.5 \text { min. arc in both directions }\end{array}$
\end{tabular}

The target for these measurements was an illuminated pinhole subtending $1 \mathrm{~min}$. arc at the eye and situated $1.8 \mathrm{~m}$. from the subject; the filters used gave maximum visibility at $4,800 \AA, 5,400 \AA$, and $6,500 \AA$ respectively.

\section{Discussion}

This movement might be taken as evidence in favour of the cluster hypothesis of retinal receptors advanced by Hartridge (1947), the eye turning so that the image of the target falls on the cluster of receptors most sensitive to the colour of the target. It will be seen that the measurements given above indicate that the red cluster of receptors should be on the temporal side of the green and blue ones, the displacements being of the same order as 
those recorded by Hartridge. Moreover, since the optical system of the eye is not achromatized and is used off axis, this distribution of clusters would agree with the coloured image of a white target as indicated in Fig. 1. In this case, however, it seems improbable that the eye would make turning movements when the colour of the target is changed; chromatic aberration alone will ensure that the image of a target of any particular colour falls on the most advantageous cluster of receptors, and hence another reason must be sought for these turning movements.

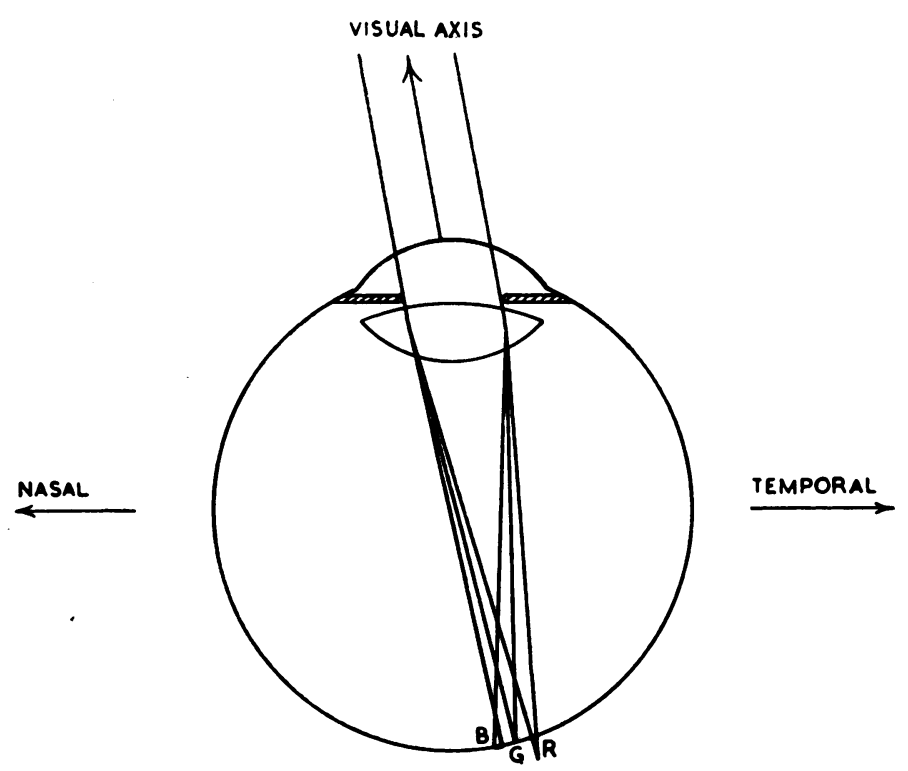

Fig. 1.-Variation of image position due to chromatic aberration only (right eye plan).

The following argument is advanced as a possible explanation of this phenomenon. The state of accommcdation of the eye changes with colour of the target and this may produce a shift of the back nodal point of the eye; since the visual axis is inclined to the opic axis of the eye, movement of the nodal point backwards or forwards will produce a lateral shift of the image on the retina as shown for the horizontal plane in Fig. 2 (opposite). In this case the eye can make turning motions to maintain the image of the target on the same point of the retina whatever the target colour. This is shown in Fig. 3 (opposite); the motion of the nodal point being towards the retina when a target in red illumination is viewed.

The angle $\alpha$ has been measured for this subject when wearing a contact lens, the method used being to find the direction in which the Purkinje images appeared most closely grouped together. This gives a value of about $5^{\circ}$ in the horizontal plane and $1.5^{\circ}$ in the vertical plane, but with the visual axis inclined downwards from the optic axis. The use of a contact lens 


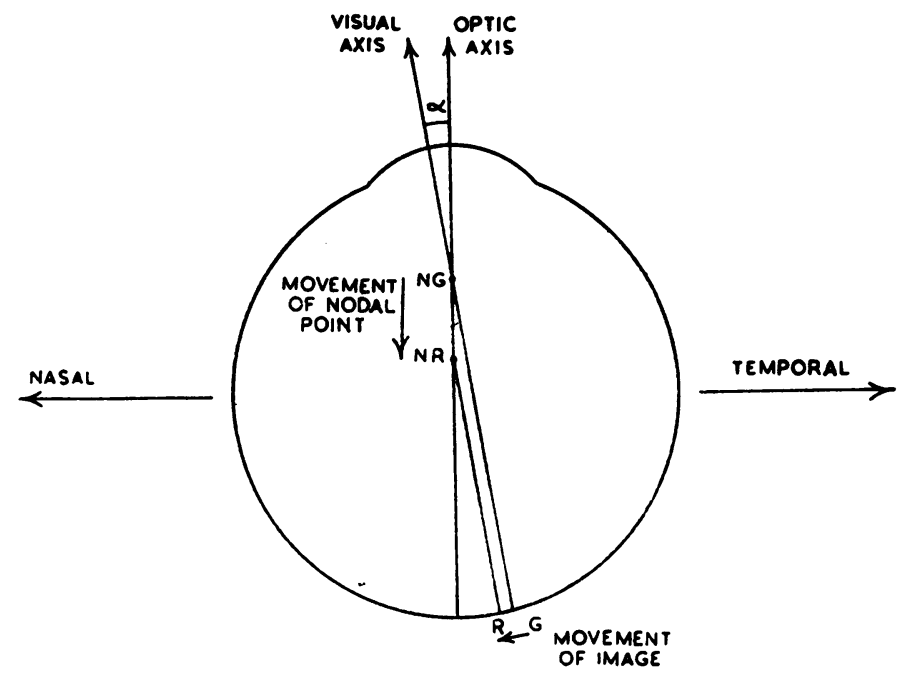

FIG. 2.-Variation of image position due to motion of nodal point as accommodation is changed (right eye plan).

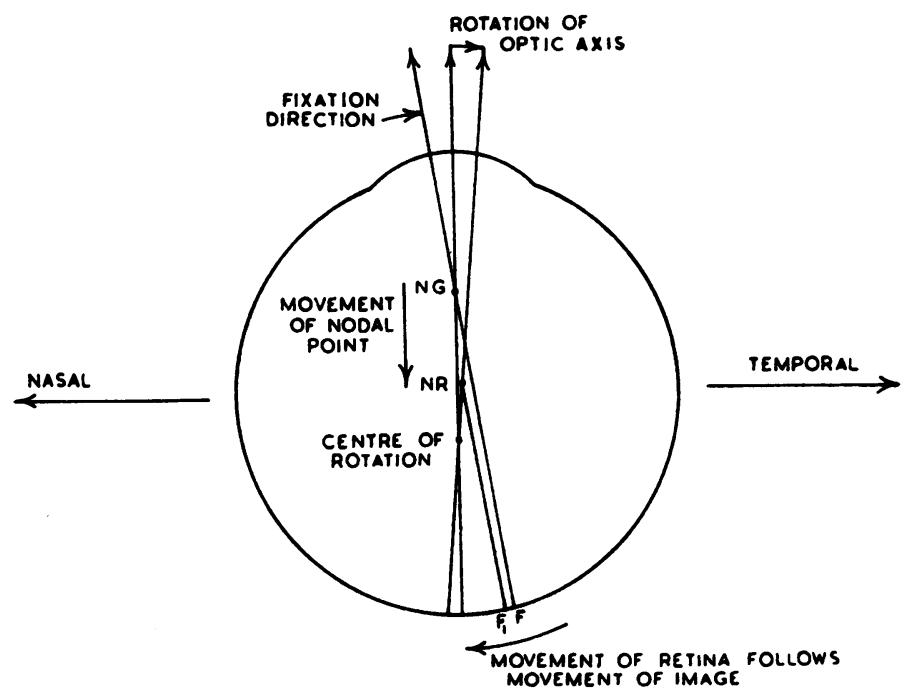

FIG. 3.-Motion of eyeball following movement of image (right eye plan).

caused the images to spread badly and the measurements could not be made with any great precision; however, in conjunction with the readings in the Table, both values for the angle $\alpha$ indicate a backward movement of the nodal point by about $0.6 \mathrm{~mm}$. when the target colour changes from green to red.

Further evidence for the motion of the nodal points can be obtained by 
plotting the size of the blind spot using a Bjerrum screen illuminated with lights of various colours.

For all subjects tested, the angular width of the blind spot was found to be about $0.5^{\circ}$ larger in red light than in green, supporting the idea that the nodal point moves towards the retina in red light; the sensitivity of the method is unfortunately not sufficient to show any significant change in the vertical direction.

This motion of the eyes may account for the anomalous effects sometimes observed in chromatic stereoscopy; for example, to some subjects red appears an advancing colour on a black background but receding on a white background. The perception of depth depends on many factors, amongst which may be listed the following:

(a) disparity of the retinal images,

(b) accommodative state of the eyes,

(c) vergence of the fixation axes.

Chromatic difference of magnification causes disparate retinal images giving red the appearance of nearness, and accommodation has to be increased when viewing a red target, giving the same impression; but as indicated by the measurements tabulated above, the divergence of the eyes increases when viewing a red target, thus leading to an impression of distance. The mental picture in any particular case possibly depends on which one (or which combination) of these cues predominates.

\section{Summary}

It has been demonstrated that a shift of the fixation direction of the eyeball occurs when the colour of the fixation target is changed. This may be due to the fact that the optical system of the eye is not achromatic, hence the position of the back nodal point is sensitive to colour; since the lens of the eye is used off-axis, a change in position of the nodal point results in the image being displaced across the retina as the object colour varies. The eyeball then makes turning movements designed to maintain the image on the same retinal receptors irrespective of target colour. This would appear to be in conflict with the cluster hypothesis advanced by Hartridge (1947). Observations on the apparent size of the blind spot in various coloured illuminations lend support to the supposition that the position of the nodal point changes with colour.

The author would like to record his thanks to Mr. R. M. Pritchard who has kindly acted as subject and to Professor R. W. Ditchburn for his encouragement and advice.

\section{REFERENCES}

DitchbuRn, R. W., and Ginsborg, B. L. (1953). J. Physiol. (Lond.), 119, 1.

FENDER, D. H. (1955). British Journal of Ophthalmology, 39, 65.

Hartridge, H. (1947). Philos. Trans. B., 232, 519. 